\title{
Two Years of Denosumab and Teriparatide Administration in Postmenopausal Women With Osteoporosis (The DATA Extension Study): A Randomized Controlled Trial
}

\author{
Benjamin Z. Leder, Joy N. Tsai, Alexander V. Uihlein, Sherri-Ann M. Burnett-Bowie, Yuli \\ Zhu, Katelyn Foley, Hang Lee, and Robert M. Neer \\ Department of Medicine, Endocrine Unit (B.Z.L., J.N.T., A.V.U., S.-A.M.B.-B., Y.Z., K.F., R.M.N.) \\ and Biostatistics Center (H.L.), Massachusetts General Hospital, Boston, Massachusetts 02114
}

\begin{abstract}
Context-Current osteoporosis medications increase bone mineral density (BMD) modestly and reduce, but do not eliminate, fracture risk. Attempts to improve efficacy by administering anabolic agents and bisphosphonates concomitantly have been unsuccessful. Conversely, 12 months of concomitant denosumab and teriparatide therapy increases BMD more than either drug alone.
\end{abstract}

Objective-The purpose of this study was to determine whether 24 months of combined denosumab and teriparatide will increase hip and spine BMD more than either individual agent.

Design-Preplanned continuation of the Denosumab and Teriparatide Administration (DATA) randomized controlled trial in which postmenopausal osteoporotic women received teriparatide ( $20 \mu \mathrm{g}$ daily), denosumab (60 mg every 6 months), or both medications for 24 months.

Participants-Participants were 94 postmenopausal women with osteoporosis.

Outcome Measures-Lumbar spine, femoral neck, total hip, and distal radius BMD and serum markers of bone turnover were measured.

Results-At 24 months, lumbar spine BMD increased more in the combination group (12.9 \pm $5.0 \%)$ than in either the teriparatide $(9.5 \pm 5.9 \%, P=.01)$ or denosumab $(8.3 \pm 3.4 \%, P=.008)$ groups. Femoral neck BMD also increased more in the combination group $(6.8 \pm 3.6 \%)$ than in either the teriparatide $(2.8 \pm 3.9 \%, P=.003)$ or denosumab $(4.1 \pm 3.8 \%, P=.008)$ groups. Similarly, total hip BMD increased more in the combination group $(6.3 \pm 2.6 \%)$ than in the teriparatide $(2.0 \pm 3.0 \%)$ or denosumab $(3.2 \pm 2.5 \%)$ groups $(P<.001$ for both $)$. Although spine and hip BMD continued to increase in the second year in all groups, these year 2 increases did not differ among groups. Serum C-telopeptide and $\mathrm{N}$-terminal propeptide of type 1 procollagen were equally suppressed in the denosumab and combination groups, whereas osteocalcin decreased more in the denosumab group than in the combination group, a difference that persisted, but lessened, in the second year of therapy.

Copyright $@ 2014$ by the Endocrine Society

Address all correspondence and requests for reprints to: Benjamin Z. Leder, Endocrine Unit, Department of Medicine, Massachusetts General Hospital, 50 Blossom Street, Thier 1051, Boston, MA 02114. bzleder@ partners.org..

Disclosure Summary: B.Z.L. has served as a consultant for Eli Lilly and Amgen. The other authors have nothing to disclose. 
Conclusions-Two years of concomitant teriparatide and denosumab therapy increases BMD more than therapy with either medication alone and more than has been reported with any current therapy. The combination of these agents may prove to be an important treatment option in patients at high risk of fracture.

Unlike treatments for the vast majority of chronic diseases, US Food and Drug Administration--approved osteoporosis treatments are currently limited to the use of a single drug at a fixed dose. Furthermore, although the therapeutic options for osteoporosis treatment have expanded greatly over the past 2 decades, no currently approved therapy is able to restore skeletal integrity in most patients with established osteoporosis. Current medications approved to treat postmenopausal osteoporosis can be separated into 2 categories. The most commonly used drugs are the antiresorptive medications such as the nitrogen-containing bisphosphonates and the receptor activator of nuclear factor $\mathrm{kB}$ ligand inhibitor denosumab. Whereas both bisphosphonates and denosumab inhibit osteoclastic bone resorption (and, to a lesser degree, bone formation), they do so by different cellular and molecular mechanisms $(1,2)$. Less commonly used and generally reserved for patients with severe and established osteoporosis are the anabolic agents PTH [PTH-(1-84)] and teriparatide [PTH-(1-34)]. These peptides potently stimulate osteoblastic bone formation but also stimulate bone resorption (3).

Attempts to combine more than 2 antiresorptive agents have demonstrated very limited additive effects on bone mass (4). Initially thought to be a more promising approach, attempts to combine PTH or teriparatide with bisphosphonates have also been unsuccessful in that no combination was shown to be consistently superior to monotherapy (5-8). Similarly, the concomitant use of PTH and the selective estrogen receptor modulator, raloxifene, also has not shown additive effects on bone mineral density (BMD) (9). In contrast, in the Denosumab and Teriparatide Administration (DATA) study, we reported that 12 months of concurrent denosumab and teriparatide increased spine and hip BMD more than either drug alone and to a greater degree than has been achieved with any currently available agent (10). The additive effect of these 2 drugs appears to be linked to the ability of denosumab to fully inhibit teriparatide-induced bone resorption but only partially inhibit teriparatide-induced bone formation. Because the effects of teriparatide on hip BMD are delayed until the second year of treatment $(7,8,11)$, however, longer-term studies are essential to confirm the superior efficacy of any teriparatide-containing approach and to ensure that any advantage of combination therapy is not transient. To test the hypothesis that sustained combination denosumab and teriparatide therapy would continue to show superior efficacy compared with mono-therapy, we prospectively extended our original DATA study to a total of 24 months of therapy and measured the changes in BMD and biochemical markers of bone turnover every 6 months.

\section{Subjects and Methods}

\section{Study subjects}

A total of 100 postmenopausal women aged 45 or older were recruited through targeted mailings, advertisements, and physician referrals. Subjects were required to be at least 36 months since last menses (or since hysterectomy if the FSH level was $>40 \mathrm{U} / \mathrm{L}$ ) and at high 
fracture risk. High fracture risk was defined as a BMD T score of $\leq-2.5$ at the spine, hip, or femoral neck, a $\mathrm{T}$ score of $\leq-2.0$ with at least one BMD independent risk factor (fracture after age 50, parental hip fracture after age 50, prior hyperthyroidism, inability to rise from a chair with arms elevated, or current smoking) (12), or a T score $\leq 1.0$ with a history of a fragility fracture. Subjects were excluded if they had evidence of hyperparathyroidism, vitamin D deficiency (serum level less than $20 \mathrm{ng} / \mathrm{mL}$ ), other congenital or acquired bone disease, history of malignancy (with the exception of nonmelanoma skin cancer), history of ionizing radiation therapy, significant cardiopulmonary, liver, or renal disease, major psychiatric disease, or excessive alcohol intake. Subjects were also excluded if they had ever taken parenteral bisphosphonates, teriparatide, or strontium ranelate. In addition, subjects were excluded if they had taken glucocorticoids or oral bisphosphonates within 6 months of enrollment or if they had taken estrogen, selective estrogen receptor modulators, or calcitonin within 3 months of enrollment.

\section{Study design}

The DATA extension study is a 12-month extension to the original 12-month, open-label, randomized controlled trial. Subjects originally randomly assigned on a 1:1:1 basis to receive $60 \mathrm{mg}$ of denosumab sc every 6 months (Prolia; Amgen, Inc), $20 \mu \mathrm{g}$ of teriparatide sc daily (Forteo; Eli Lilly, Inc), or both medications for 12 months continued their assigned treatment for an additional 12 months (24 months of total therapy). Before the initial randomization, subjects were stratified based on age ( $<65$ or $\ 65$ years) and prior bisphosphonate use (yes/no). Subjects were seen at $0,3,6,12,18$, and 24 months, and blood sampling and dual-energy x-ray absorptiometry (DXA) were performed at each visit. All blood sampling was done before teriparatide administration (ie, 24 hours after the last teriparatide dose), and physicians interpreting the BMD assessments were blinded to the treatment group. Subjects were given calcium carbonate and vitamin D supplements if needed to achieve a total daily intake of $1200 \mathrm{mg}$ of elemental calcium and to maintain a serum 25-hydroxyvitamin D level of at least $20 \mathrm{ng} / \mathrm{mL}$. Adherence to teriparatide was assessed by subject diary. All subjects provided written informed consent. The study was approved by the Partners Healthcare Institutional Review Board and is registered with ClinicalTrials.gov (number NCT00926380).

\section{BMD measurements}

Areal BMD of the posterior-anterior lumbar spine (PA spine), total hip, femoral neck, and distal one-third radius shaft was measured by DXA using a QDR 4500A densitometer (Hologic). All scans of an individual subject were performed on the same densitometer. Quality control measurements were performed daily with a Hologic anthropomorphic spine phantom. Our SDs of in vivo same-day reproducibility are $0.005,0.006$, and $0.007 \mathrm{~g} / \mathrm{cm}^{2}$ for PA spine, total hip, and femoral neck BMD measurements, respectively.

\section{Biochemical measurements}

Fasting morning blood samples (collected 24 hours after last injection for patients taking teriparatide) were obtained at each visit. Serum osteocalcin (OC) was measured via electrochemiluminescent immunoassay (Meso Scale Discovery) with inter- and intra-assay coefficients of variation (CVs) of $10 \%$ and $8 \%$, respectively. Serum amino-terminal 
propeptide of type 1 procollagen (P1NP) was measured via RIA (Orion Diagnostica) with inter- and intra-assay CVs of $6 \%$ to $10 \%$ and $7 \%$ to $10 \%$, respectively. Serum $\beta$-C-terminal telopeptide of type 1 collagen (CTX) was measured via double-antibody ELISA (Roche Diagnostics) with inter- and intra-assay CVs of $2 \%$ to $4 \%$ and $18 \%$, respectively.

Biochemical markers of bone turnover were only measured in subjects completing 24 months of therapy $(n=83)$. For each marker, all blood samples from a participant were analyzed together in the same assay run.

\section{Statistical analysis}

We used a modified intention-to-treat analysis, which included all data from subjects completing at least 1 postbaseline visit. Baseline characteristics were compared by one-way ANOVA. The predetermined primary endpoint was the percent change in PA spine BMD at 24 months. Secondary endpoints included the percent change in total hip, femoral neck, and radius shaft BMD as well as the overall change in serum OC, P1NP, and CTX. Betweengroup differences in the mean change in BMD from baseline to 24 months were examined by one-way ANOVA, and subsequent between-group differences were confirmed by an independent-samples $t$ test. Between-group differences in the change in BMD from 12 to 24 months were also examined by one-way ANOVA with subsequent between-group differences confirmed by an independent-samples $t$ test. Within-group changes were assessed by a paired $t$ test. The area under the curve (AUC) from the baseline to 24 months was calculated in each group for each bone turnover marker. Between-group differences in AUC were then compared by the same method described above as was the change in the marker level at each time point. Two-sided $P$ values of $\leq 05$ were considered significant.

\section{Results}

Of the 100 enrolled women, 94 completed at least 1 post-baseline visit and are included in this analysis; 83 women completed all visits (Figure 1). There were no significant differences in the baseline characteristics among the 3 treatment groups (Table 1). In addition, there were no significant differences in the baseline characteristics of women completing all visits compared with those of the full cohort. Because a study physician administered denosumab, adherence was $100 \%$. All women in the combination groups and $92 \%$ of women in the teriparatide monotherapy group reported taking at least $85 \%$ of their prescribed teriparatide.

\section{BMD}

Figure 2 shows the changes in DXA-derived areal BMD over the 24-month treatment period. After 24 months of treatment, mean PA spine BMD increased significantly in all treatment groups relative to the baseline ( $P<.0001$ for all within-group comparisons). Spine BMD increased more in the combination group $(12.9 \pm 5.0 \%)$ than in the teriparatide $(9.5 \pm$ $5.9 \%)$ or denosumab $(8.3 \pm 3.4 \%)$ groups $(P=.01$, combination vs teriparatide; $P=.008$, combination vs denosumab). The increase in spine BMD did not differ significantly between the teriparatide and denosumab groups $(P=.73)$. 
Femoral neck BMD also increased significantly in all groups between months 0 and 24 relative to the baseline ( $P<.0008$ for all within group comparisons). Femoral neck BMD increased more between months 0 and 24 in the combination group $(6.8 \pm 3.6 \%)$ than in the teriparatide $(2.8 \pm 3.9 \%)$ or denosumab $(4.1 \pm 3.8 \%)$ group $(P=.003$, combination vs teriparatide; $P=.008$ combination vs denosumab). The increase in femoral neck BMD did not differ significantly between the teriparatide and denosumab groups $(P=.23)$.

Similarly, after 24 months of treatment, total hip BMD increased in all groups relative to the baseline ( $P<.001$ for the denosumab and combination groups; $P=.002$ for the teriparatide group). Total hip BMD increased more between months 0 and 24 in the combination group $(6.3 \pm 2.6 \%)$ than in the teriparatide $(2.0 \pm 3.0 \%)$ or denosumab $(3.2 \pm 2.5 \%)$ group $(P<$. 001 , combination vs teriparatide and combination vs denosumab). The increase in total hip BMD did not differ significantly between the teriparatide and denosumab groups $(P=.08)$.

BMD at the distal radius increased by $2.1 \pm 3.1 \%$ in the denosumab group and by $2.2 \pm$ $3.1 \%$ in the combination group ( $P<.01$ for both comparisons vs baseline). The small decrease in BMD in the teriparatide group $(-1.7 \pm 4.6 \%)$ was not statistically significant $(P$ $=.09$ ). The magnitude of the change in distal radius BMD was similar in the denosumab and combination groups $(P=.95)$, although both groups differed significantly compared with the teriparatide group $(P<.004$ for both groups vs teriparatide).

There were no significant between-group differences in the BMD changes in the second year of therapy (Figure 3). There were, however, significant within-group increases in BMD between months 12 and 24. Specifically, spine BMD increased by $2.6 \pm 3.7 \%$ between months 12 and 24 in the teriparatide group, $2.7 \pm 2.2 \%$ between months 12 and 24 in the denosumab group, and $3.3 \pm 3.0 \%$ between months 12 and 24 in the combination group ( $P$ $<.002$ for all within-group comparisons). Over the same interval, femoral neck BMD increased by $2.4 \pm 4.8 \%$ in the teriparatide group, $1.8 \pm 3.5 \%$ in the denosumab group, and $1.6 \pm 2.6 \%$ in the combination group ( $P<.05$ for all within-group comparisons), whereas total hip BMD increased by $1.5 \pm 2.6 \%$ in the teriparatide group $(P=.007), 0.6 \pm 2.0 \%$ in the denosumab group $(P=.09)$, and $1.3 \pm 2.3 \%$ in the combination group $(P=.01)$.

\section{Biochemical markers of bone turnover}

Figure 4 shows the mean percent change in serum OC, P1NP, and CTX during the 24-month treatment period (the teriparatide group is shown separately for clarity). In women treated with teriparatide alone, mean serum OC, P1NP, and CTX increased significantly at all time points, whereas they decreased at all time points in the other 2 groups $(P<.0001$ for all within-group comparisons and between-group comparisons in net AUC and each individual time point). In the teriparatide group, the increase in these markers appeared to peak between months 6 and 12 and then reverted toward baseline between months 12 and 24 .

In the denosumab group, serum OC, which had decreased by $55 \pm 20 \%$ at month 12 , remained similarly suppressed at months 18 and 24. In contrast, in the combination group, OC decreased more slowly. At month 12, OC had decreased by $39 \pm 22 \%$ and at month 24 had decreased by $48 \pm 25 \%$. Overall mean serum OC (net AUC) was lower in the denosumab monotherapy group than in the combination group $(P<.001)$. Furthermore, OC 
levels remained lower in the denosumab group than in the combination group at all time points $(P<.002)$ despite progressively greater suppression in the combination group in year 2. Specifically, the difference in the percent reduction between the denosumab and combination groups decreased from $16 \%$ at month 12 to $8 \%$ at month 24 . Serum P1NP, which had decreased similarly in the denosumab and combination groups at month 12 remained similarly suppressed thereafter $(-59 \pm 28 \%$ and $-65 \pm 18 \%$, respectively). The overall mean serum P1NP (net AUC) was similar in the denosumab and combination groups as it was at each measured time point (not significant for all comparisons). Finally, serum CTX, which decreased similarly in both the denosumab and combination groups at month 12 , remained similarly suppressed at month $24(-49 \pm 40 \%$ and $-57 \pm 33 \%$, respectively, not significant for the between-group comparison in net AUC and the between-group comparison at each time point).

\section{Safety}

Mild asymptomatic hypercalcemia (>10.8 mg/dL) was detected rarely in year 1 as reported previously (10). In year 2 , no calcium levels $>10.8 \mathrm{mg} / \mathrm{dL}$ were observed. Serious adverse events occurring in year 1 were also reported previously (10). Serious adverse events occurring in year 2 included a subject with cholecystitis requiring cholecystectomy (denosumab group) and a subject with diverticulitis requiring sigmoid colectomy (teriparatide group). All serious adverse events, including those occurring in year 1, were considered unrelated to the study therapy, as assessed by study investigators and an independent safety monitoring board.

\section{Discussion}

In this 24-month randomized controlled trial, we demonstrated that the combination of denosumab and teriparatide increases BMD at the femoral neck, total hip, and PA spine significantly more than either drug alone. Moreover, the increases observed in the combination group (spine $=12.9 \%$, femoral neck $=6.8 \%$, and total hip $=6.3 \%$ ) are greater than increases that can be achieved with any currently available treatment $(11,13-18)$. These findings are consistent with various animal models, including the ovariectomized rat in which the combination of osteoprotegerin (an endogenous molecule with properties similar to those of denosumab) and teriparatide greatly reduced osteoclast number, did not reduce osteoblast number, and increased trabecular and cortical BMD more than either agent alone (19). Similarly, in a mouse model in which osteoprotegerin and teriparatide were coadministered, the increase in femoral BMD was additive, and the increase in spine BMD exceeded the cumulative changes with each agent individually (20).

These findings continue to contrast with those of clinical studies investigating the effects of combined teriparatide and bisphosphonates. For example, in a prior 30-month clinical trial in which postmenopausal women were randomly assigned to receive either $10 \mathrm{mg}$ of alendronate daily, $40 \mu \mathrm{g}$ of teriparatide daily, or both medications (teriparatide was started at month 6), DXA-derived spine and hip BMD increased more in women treated with teriparatide alone than in those treated with alendronate alone or with both medications (8). Also in contrast to the current study, biochemical markers of bone resorption were 
suppressed less in subjects receiving both alendro-nate and teriparatide than in subjects receiving alendro-nate alone (8). Similar findings were reported when the identical study was performed in men (7). Additional 2-year studies investigating the effects of other bisphosphonates in combination with teriparatide are lacking, although it was recently reported that women treated with 9 months of teriparatide followed by 9 months of teriparatide plus alendronate experienced greater BMD increases at the hip than women treated with 18 months of teriparatide alone (there was also a trend toward greater BMD increases at the spine) (21).

By extending the current study to 2 years, we are now able to more definitively assess the comparative efficacy of the denosumab [H11001] teriparatide treatment approach. This is especially pertinent because teriparatide does not appreciably increase hip and femoral neck BMD in the first year of therapy but does so in the second year of therapy (as is demonstrated in the teriparatide monotherapy group in the current study as well as in prior teriparatide clinical trials) $(7,8,11,22,23)$. It is also important to note that the BMD changes in the second year of therapy in the current study were generally similar in all treatment groups at all anatomic sites. This latter finding may be explained by the shrinking (but still significant) gap in denosumab and the ability of combination therapy to inhibit bone resorption between months 12 and 24. This observation also raises the possibility that the most cost-effective way to achieve greater increases in BMD may be to treat with combined teriparatide and denosumab for 1 year followed by an antiresorptive agent alone for the second year. This hypothesis deserves evaluation.

There are several potential limitations to our study. First, the study was powered to detect changes in BMD but not changes in fracture rates. A study aimed to assess comparative fracture risk reduction would require a very large sample size and would not be feasible in the context of an investigator-initiated trial. Nonetheless, treatment-induced increases in $\mathrm{BMD}$ at the various anatomic sites have proven to be reliable, although not perfect, surrogates for efficacy in fracture prevention and the larger increases in BMD in the combination group compared with those in the monotherapy groups (ranging from $3.4 \%$ to $5.6 \%$ at the spine and $3.1 \%$ to $4.3 \%$ at the hip) are very likely to be clinically significant. In addition, although treatment-induced improvements in the non-BMD determinants of bone strength may be important, BMD changes in patients treated with both denosumab and teriparatide mono-therapy have been shown to predict fracture reduction $(24,25)$. Furthermore, our cohort size was limited and made up predominately of white women, potentially limiting generalizability and making subgroup analysis not feasible.

It is also notable that there are small nonstatistically significant differences in the rate, duration, and washout period of prior bisphosphonate use among the 3 treatment groups. Further analysis of our data (not shown), however, demonstrates no difference in the response to therapy in subjects with or without a history of bisphosphonate exposure (both overall and within each group), and the between-group relationships are unchanged if all subjects exposed to bisphosphonates are removed from the analysis.

In summary, we have shown that 24 months of combined denosumab and teriparatide therapy increases BMD at the spine, hip, and femoral neck more than either drug alone and 
more than any currently available agent. Although a larger study would be needed to definitively demonstrate a fracture-reduction benefit, the sustained 2-year superiority on BMD accrual strongly supports the conclusion that this particular combined approach may be a useful option in patients at very high risk of fragility fracture.

\section{Acknowledgments}

We thank Dr John Potts for his consistent advice and support, the staff at the Massachusetts General Hospital Bone Density Center, and our dedicated study volunteers.

This work was supported by Eli Lilly Inc, Amgen Inc, and by the Harvard Clinical and Translational Science Center, the National Center for Research Resources (Grant 1 ULI RR025758-04). Neither Eli Lilly nor Amgen had any role in study design or data analysis or data interpretation.

\section{Abbreviations}

$\begin{array}{ll}\text { AUC } & \text { area under the curve } \\ \text { BMD } & \text { bone mineral density } \\ \text { CTX } & \beta \text {-C-terminal telopeptide of type 1 collagen } \\ \text { CV } & \text { coefficient of variation } \\ \text { DXA } & \text { dual-energy x-ray absorptiometry } \\ \text { OC } & \text { osteocalcin } \\ \text { P1NP } & \text { propeptide of type 1 procollagen } \\ \text { PA spine } & \text { posterior-anterior lumbar spine }\end{array}$

\section{References}

1. Reszka AA, Rodan GA. Mechanism of action of bisphosphonates. Curr Osteoporos Rep. 2003; 1:45-52. [PubMed: 16036064]

2. Delmas PD. Clinical potential of RANKL inhibition for the management of postmenopausal osteoporosis and other metabolic bone diseases. J Clin Densitom. 2008; 11:325-338. [PubMed: 18375161]

3. Dempster DW, Zhou H, Recker RR, et al. Skeletal histomorphometry in subjects on teriparatide or zoledronic acid therapy (SHOTZ) study: a randomized controlled trial. J Clin Endocrinol Metab. 2012; 97:2799-2808. [PubMed: 22701017]

4. Compston J. The use of combination therapy in the treatment of postmenopausal osteoporosis. Endocrine. 2012; 41:11-18. [PubMed: 22038453]

5. Black DM, Greenspan SL, Ensrud KE, et al. The effects of parathyroid hormone and alendronate alone or in combination in post-menopausal osteoporosis. N Engl J Med. 2003; 349:1207-1215. [PubMed: 14500804]

6. Cosman F, Eriksen EF, Recknor C, et al. Effects of intravenous zoledronic acid plus subcutaneous teriparatide [rhPTH(1-34)] in postmenopausal osteoporosis. J Bone Miner Res. 2011; 26:503-511. [PubMed: 20814967]

7. Finkelstein JS, Hayes A, Hunzelman JL, Wyland JJ, Lee H, Neer RM. The effects of parathyroid hormone, alendronate, or both in men with osteoporosis. N Engl J Med. 2003; 349:1216-1226. [PubMed: 14500805]

8. Finkelstein JS, Wyland JJ, Lee H, Neer RM. Effects of teriparatide, alendronate, or both in women with postmenopausal osteoporosis. J Clin Endocrinol Metab. 2010; 95:1838-1845. [PubMed: 20164296] 
9. Deal C, Omizo M, Schwartz EN, et al. Combination teriparatide and raloxifene therapy for postmenopausal osteoporosis: results from a 6-month double-blind placebo-controlled trial. J Bone Miner Res. 2005; 20:1905-1911. [PubMed: 16234962]

10. Tsai JN, Uihlein AV, Lee H, et al. Teriparatide and denosumab, alone or combined, in women with postmenopausal osteoporosis: the DATA study randomised trial. Lancet. 2013; 382:50-56. [PubMed: 23683600]

11. Neer RM, Arnaud CD, Zanchetta JR, et al. Effect of parathyroid hormone (1-34) on fractures and bone mineral density in postmenopausal women with osteoporosis. N Engl J Med. 2001; 344:1434-1441. [PubMed: 11346808]

12. Black DM, Steinbuch M, Palermo L, et al. An assessment tool for predicting fracture risk in postmenopausal women. Osteoporos Int. 2001; 12:519-528. [PubMed: 11527048]

13. Black DM, Delmas PD, Eastell R, et al. Once-yearly zoledronic acid for treatment of postmenopausal osteoporosis. N Engl J Med. 2007; 356:1809-1822. [PubMed: 17476007]

14. Cummings SR, San Martin J, McClung MR, et al. Denosumab for prevention of fractures in postmenopausal women with osteoporosis. N Engl J Med. 2009; 361:756-765. [PubMed: 19671655]

15. Liberman UA, Weiss SR, Bröll J, et al. Effect of oral alendronate on bone mineral density and the incidence of fractures in postmenopausal osteoporosis. The Alendronate Phase III Osteoporosis Treatment Study Group. N Engl J Med. 1995; 333:1437-1443. [PubMed: 7477143]

16. McClung MR, Geusens P, Miller PD, et al. Effect of risedronate on the risk of hip fracture in elderly women. Hip Intervention Program Study Group. N Engl J Med. 2001; 344:333-340. [PubMed: 11172164]

17. Chesnut CH III, Skag A, Christiansen C, et al. Effects of oral ibandronate administered daily or intermittently on fracture risk in post-menopausal osteoporosis. J Bone Miner Res. 2004; 19:12411249. [PubMed: 15231010]

18. Ettinger B, Black DM, Mitlak BH, et al. Reduction of vertebral fracture risk in postmenopausal women with osteoporosis treated with raloxifene: results from a 3-year randomized clinical trial. Multiple Outcomes of Raloxifene Evaluation (MORE) Investigators. JAMA. 1999; 282:637-645. [PubMed: 10517716]

19. Kostenuik PJ, Capparelli C, Morony S, et al. OPG and PTH-(1-34) have additive effects on bone density and mechanical strength in osteopenic ovariectomized rats. Endocrinology. 2001; 142:4295-4304. [PubMed: 11564687]

20. Samadfam R, Xia Q, Goltzman D. Co-treatment of PTH with osteoprotegerin or alendronate increases its anabolic effect on the skeleton of oophorectomized mice. J Bone Miner Res. 2007; 22:55-63. [PubMed: 17014384]

21. Muschitz C, Kocijan R, Fahrleitner-Pammer A, Lung S, Resch H. Antiresorptives overlapping ongoing teriparatide treatment result in additional increases in bone mineral density. J Bone Miner Res. 2013; 28:196-205. [PubMed: 22836585]

22. Kurland ES, Cosman F, McMahon DJ, Rosen CJ, Lindsay R, Bilezikian JP. Parathyroid hormone as a therapy for idiopathic osteoporosis in men: effects on bone mineral density and bone markers. J Clin Endocrinol Metab. 2000; 85:3069-3076. [PubMed: 10999788]

23. Lindsay R, Nieves J, Formica C, Henneman E, Woelfert L, Shen V, Dempster D, Cosman F. Randomised controlled study of effect of parathyroid hormone on vertebral-bone mass and fracture incidence among postmenopausal women on oestrogen with osteoporosis. Lancet. 1997; 350:550-555. [PubMed: 9284777]

24. Chen P, Miller PD, Delmas PD, Misurski DA, Krege JH. Change in lumbar spine BMD and vertebral fracture risk reduction in teriparatide-treated postmenopausal women with osteoporosis. $\mathrm{J}$ Bone Miner Res. 2006; 21:1785-1790. [PubMed: 17002571]

25. Austin M, Yang YC, Vittinghoff E, et al. Relationship between bone mineral density changes with denosumab treatment and risk reduction for vertebral and nonvertebral fractures. J Bone Miner Res. 2012; 27:687-693. [PubMed: 22095631] 


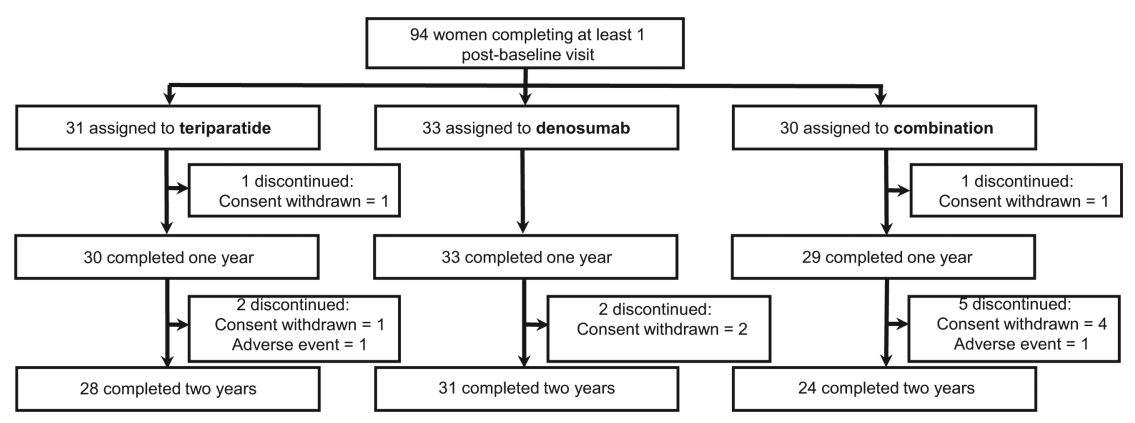

figure 1.

subject disposition. 

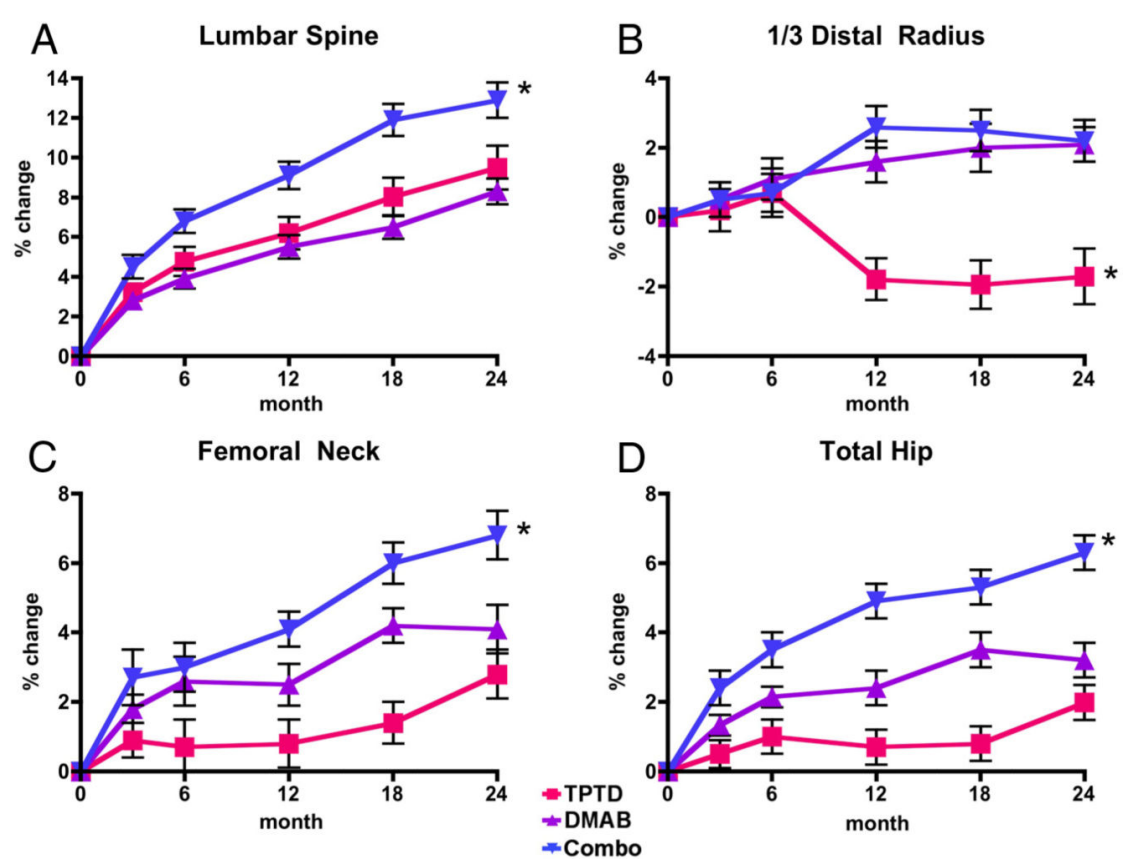

Figure 2.

Mean percent change (SEM) in BMD from baseline to 24 months in the lumbar spine (A), one-third distal radius (B), femoral neck (C), and total hip (D) in the teriparatide (TPTD), denosumab (DMAB), and combination (Combo) groups. ${ }^{*}, P<.05$ compared with other groups. 

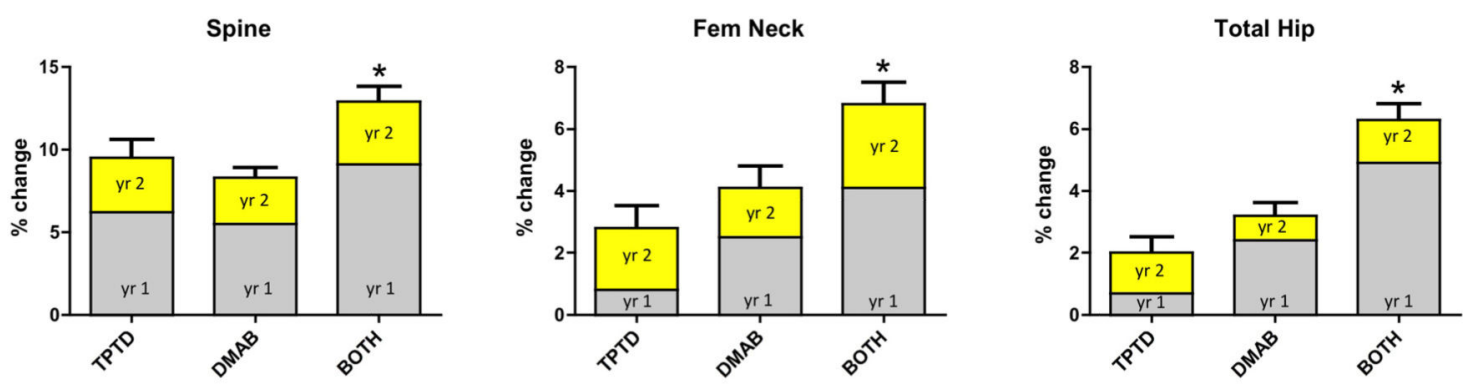

Figure 3.

Mean percent change (SEM) in BMD (grams per square centimeter) from months 0 to 12 (gray) and months 12 to 24 (yellow) in the teriparatide (TPTD), denosumab (DMAB), and combination (Combo) groups. $*, P<.05$ vs other groups for the overall 0 to 24 month change. Changes between 12 and 24 months did not differ significantly among groups. 

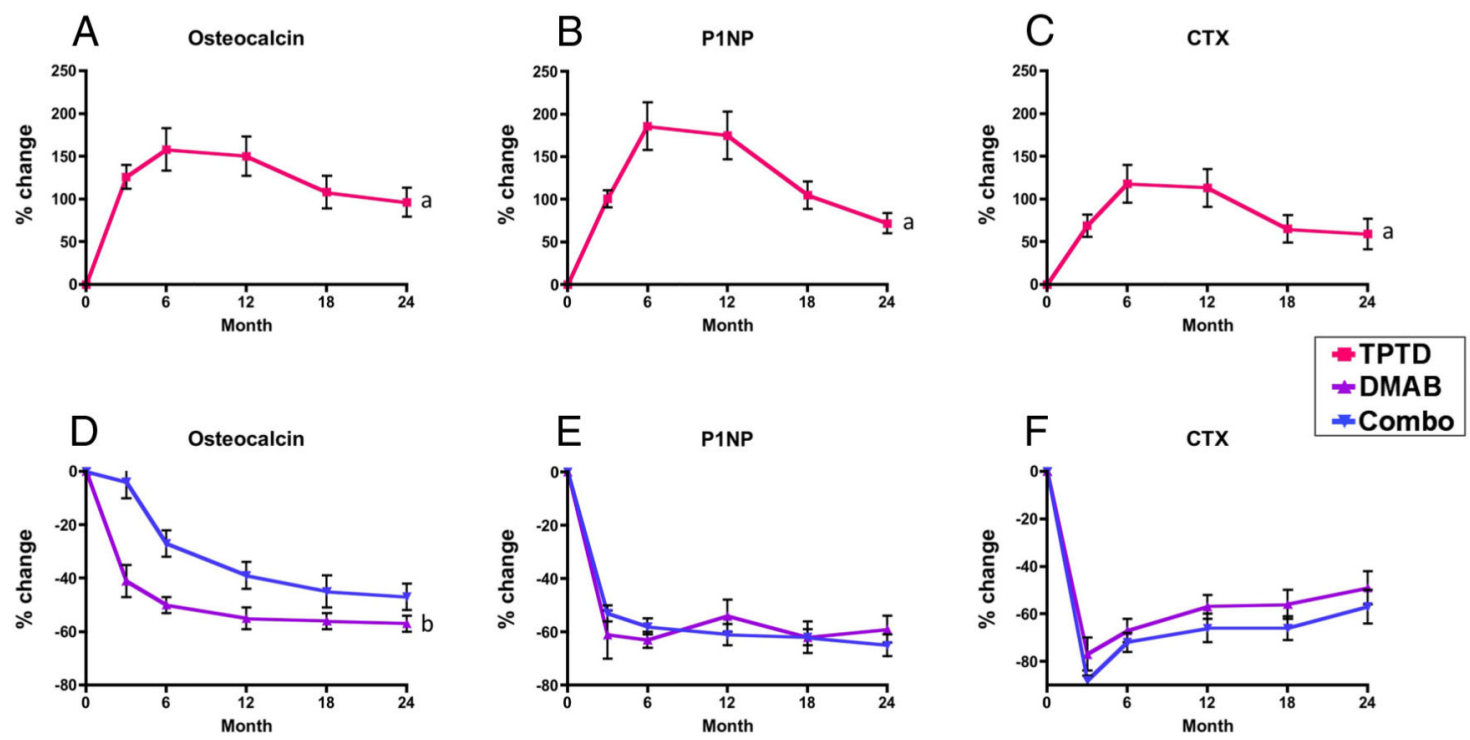

Figure 4.

Markers. Mean percent change (SEM) in bone turnover markers from baseline to 24 months in the teriparatide (TPTD, A-C) and denosumab (DMAB) and combination (Combo) groups (D-F). ${ }^{\mathrm{a}}, P<.0001 \mathrm{vs}$ denosumab and combination at all time points. ${ }^{\mathrm{b}}, P<.005 \mathrm{vs}$ denosumab at all time points. Data for the teriparatide group and other groups are graphed separately for figure clarity. Error bars that are not seen are contained within the symbols. 
Table 1

Baseline Characteristics of All Women in the Modified Intention-to-Treat Analysis

\begin{tabular}{|c|c|c|c|c|}
\hline Characteristic & Teriparatide $(\mathbf{n}=\mathbf{3 1})$ & Denosumab $(n=33)$ & Combination $(n=30)$ & $P$ Value \\
\hline Age, y & $65.5 \pm 7.9$ & $66.3 \pm 8.3$ & $65.9 \pm 9.0$ & .94 \\
\hline Body mass index, $\mathrm{kg} / \mathrm{m}^{2}$ & $25.5 \pm 3.8$ & $24.1 \pm 3.9$ & $25.4 \pm 4.9$ & .31 \\
\hline White, non-Hispanic (\%) & $31(100)$ & $30(91)$ & $27(90)$ & .20 \\
\hline History of fracture, n (\%) & $16(52)$ & $12(36)$ & $10(33)$ & .29 \\
\hline Previous bisphosphonate use, n (\%) & $13(42)$ & $12(36)$ & $10(33)$ & .78 \\
\hline Duration of use, months & $40 \pm 25$ & $43 \pm 27$ & $28 \pm 21$ & .31 \\
\hline Time since discontinuation, months & $27 \pm 20$ & $36 \pm 23$ & $42 \pm 17$ & .24 \\
\hline 25-Hydroxyvitamin $\mathrm{D}, \mathrm{ng} / \mathrm{mL}$ & $31.2 \pm 8.5$ & $35.3 \pm 10.5$ & $33.9 \pm 11.8$ & .28 \\
\hline Alkaline phosphatase, U/L & $75.8 \pm 16.8$ & $78.8 \pm 16.8$ & $84.2 \pm 20.8$ & .20 \\
\hline $\mathrm{OC}, \mathrm{ng} / \mathrm{mL}$ & $49.0 \pm 28.8$ & $42.9 \pm 19.4$ & $52.2 \pm 29.9$ & .37 \\
\hline $\mathrm{P} 1 \mathrm{NP}, \mu \mathrm{g} / \mathrm{L}$ & $46.0 \pm 19.5$ & $45.7 \pm 16.7$ & $49.3 \pm 20.9$ & .72 \\
\hline CTX, ng/mL & $0.36 \pm 0.15$ & $0.39 \pm 0.21$ & $0.43 \pm 0.17$ & .31 \\
\hline PA spine BMD, $\mathrm{g} / \mathrm{cm}^{2}$ & $0.823 \pm 0.111$ & $0.866 \pm 0.088$ & $0.856 \pm 0.131$ & .31 \\
\hline Femoral neck BMD, $\mathrm{g} / \mathrm{cm}^{2}$ & $0.643 \pm 0.061$ & $0.641 \pm 0.086$ & $0.642 \pm 0.067$ & .99 \\
\hline Total hip BMD, $\mathrm{g} / \mathrm{cm}^{2}$ & $0.757 \pm 0.068$ & $0.766 \pm 0.100$ & $0.759 \pm 0.073$ & .94 \\
\hline One-third radius $\mathrm{BMD}, \mathrm{g} / \mathrm{cm}^{2}$ & $0.612 \pm 0.069$ & $0.602 \pm 0.082$ & $0.613 \pm 0.070$ & .84 \\
\hline
\end{tabular}

Values are means \pm SD. 